Ann. Zootech., I973, 22 (4), 50I-504.

NOTE

\title{
COMPARAISON DE DIVERS CRITÈRES POUR ÉVALUER LA VALEUR DES PROTÉINES DU RÉGIME DES PONDEUSES
}

\author{
J. GUILlaUmE et C. CALET \\ Station de Recherches avicoles \\ Centre de Recherches de Tours, I. N. R. A., \\ Nouzilly, B.P. 1, 37380 Monnaie
}

On admet classiquement que la valeur des différents critères qui caractérisent la production d'une poule n'est pas affectée de la même façon lorsque la composition azotée du régime change. Les critères les plus souvent étudiés sont le nombre des œufs pondus et le poids de l'œuf. Plus récemment, Moreno et al., 1970 ont fait appel à la longueur des séries tandis que Larbier, Blum, Guillaume, 1972 y ont ajouté le poids du vitellus et de l'albumen. Ce sont autant de paramètres qui concernent directement la ponte. Nous avons jugé opportun d'y ajouter deux nouveaux critères, la durée et la fréquence des pauses qui caractérisent la ponte. de manière indirecte, à partir des jours où la poule ne pond pas.

Quels que soient ces critères, on ignore ceux qui sont capables de caractériser au mieux (sensibilité et spécificité) un facteur nutritionnel donné. Dans ce but nous avons comparé $x 7$ paramètres différents en portant une attention particulière sur leur variabilité. Les régimes alimentaires diffèrent par leur teneur en acides aminés, mais leur composition est voisine en vue d'éprouver la sensibilité des critères étudiés.

\section{MATÉRIEL, ETT MÉTHODES}

Nous disposons de 3 lots homogènes de 24 poules à performances élevées (croisement de type Rhode Island Red, Warren-Studler) réparties dans les cages d'une salle conditionnée où sont maintenues constantes la température $\left(15^{\circ} \mathrm{C} \pm \mathrm{I}\right)$ et l'hygrométrie (70 p. I00 \pm 5$)$. L'éclairage est également constant ( 15 h par jour). L'âge des poules est de 23 semaines au début de l'essai, époque où le taux de ponte atteint $25 \mathrm{p}$. Ioo environ. L'essai dure I 40 jours et est décomposé en 5 périodes successives de 28 jours.

Trois régimes sont constitués; leurs caractéristiques sont peu différentes et portent sur la composition en acides aminés. Le régime témoin ( $\mathrm{T}$ ) est complet et équilibré ( $\mathrm{I} 6,8$ p. roo de protéines et $0,3 \mathrm{I}$ p. roo de méthionine). Le second (MP) est légèrement carencé en protéines 
( I 4,9 p. IOO). Sa déficience concerne l'ensemble des acides aminés; en particulier sa teneur en méthionine est de 0,26 p. Ioo. Le troisième (M) a le même taux protidique que le témoin mais il est carencé en méthionine seulement $(0,26 \mathrm{p}$. Ioo comme dans le régime MP).

Les caractéristiques des trois aliments sont rassemblées dans le tableau $\mathrm{I}$.

\section{TABLEAU I}

\begin{tabular}{c|c|c|c|c|c|c|c}
\hline \hline Régimes & $\begin{array}{c}\text { Énergie } \\
\text { métabolis. } \\
\text { (kcal/g) }\end{array}$ & $\begin{array}{c}\text { Protéines } \\
\mathrm{N} \times 6,25 \\
(\mathrm{p} .100)\end{array}$ & $\begin{array}{c}\text { Méthionine } \\
\text { (p. 100) }\end{array}$ & $\begin{array}{c}\text { Méthionine } \\
+ \text { Cystine } \\
\text { (p. 100) }\end{array}$ & $\begin{array}{c}\text { Lysine } \\
\text { (p. 100) }\end{array}$ & $\begin{array}{c}\text { Ca } \\
\text { (p. 100) }\end{array}$ & $\begin{array}{c}\mathrm{P} \\
\text { disponible } \\
\text { (p. 100) }\end{array}$ \\
\hline $\mathrm{T}$ & $-2,76$ & 16,8 & 0,31 & 0,66 & 0,80 & 3,1 & 0,35 \\
\hline $\mathrm{M}$ & $-2,75$ & 16,8 & 0,26 & 0,60 & 0,80 & 3,1 & 0,35 \\
\hline $\mathrm{MP}$ & 2,75 & 14,9 & 0,26 & 0,57 & 0,80 & 3,1 & 0,35 \\
\hline \hline
\end{tabular}

Les poules et les quantités d'aliment qu'elles consomment sont pesées à la fin des périodes. Tous les cufs sont collectés et pesés individuellement. Pendant une semaine de chaque mois, les oufs sont cassés, le vitellus et la coquille sont pesés; le poids de l'albumen est obtenu par différence. On apprécie la vitellogenèse et l'albuminogenèse de chaque poule par les quantités de jaune et de blanc exportées dans l'œuf au cours de chaque période. On multiplie pour cela le nombre des œufs de la période par les poids moyens respectifs du vitellus et de l'albumen établis pendant la semaine où ils ont été mesurés.

Les critères étudiés comprennent : mortalité, consommation, gain de poids des animaux, intensité de ponte, poids moyen de l'œuf, indice de consommation, ainsi que poids moyen et production quotidienne d'albumen et de vitellus. Nous y avons ajouté la longueur des séries proposées par Harms et WALDROUP, 1963 et d'autres critères peu ou pas étudiés tels que la fréquence des séries, la durée et la fréquence des pauses durant de 2 à 4 jours.

On soumet à l'analyse de variance d'une part les moyennes individuelles pour l'ensemble de l'essai, et d'autre part, les moyennes individuelles pour chaque période à l'exception de la première pendant laquelle la ponte était encore faible. N'entrent pas dans cette dernière analyse les critères relatifs aux séries, qui n'ont de signification que pour l'essai entier. L'indice de consommation n'a pas été soumis à l'analyse statistique étant données les faibles différences observées d'une part et la difficulté de comparer des rapports de variables aléatoires d'autre part.

\section{RÉSULTATS ET DISCUSSION}

L'ensemble des résultats est rassemblé dans le tableau 2.

Comme nous l'avions souhaité, aucun régime ne s'avère franchement déficient puisque le taux de ponte est élevé dans les trois lots. Notons que la production du lot MP est légèrement plus faible que celle des deux autres, mais la différence n'est pas significative. Parmi les critères étudiés, certains comme le gain de poids des poules, l'intensité de ponte, la longueur de la série, la fréquence des séries ne diffèrent pas significativement. Ils ont d'ailleurs une variabilité considérable qui les rend inutilisables sur de petits effectifs et vraisemblablement peu précis sur des lots plus importants. En revanche, deux critères jusqu'alors ignorés devraient retenir l'attention : ce sont la durée des pauses et la fréquence des pauses de 2 à 4 jours. L'une et l'autre permettent de déceler la déficience en protéine du régime sans que l'on puisse affirmer que ces mesures sont très utilisables. En effet, le petit nombre de sujets étudiés n'a pas permis de juger de la normalité de la distribution de toutes les variables aléatoires citées plus haut. 
Des différences apparaissent encore plus nettement quand on s'adresse aux caractéristiques de l'œuf ; elles ont trait à la fois aux valeurs moyennes de ces dernières et à leur variabilité. Pour l'essai entier, le poids moyen de l'œuf et de ses constituants, exception faite de la coquille, permet de mettre mieux en évidence des différences entre les régimes que les productions journalières d'œuf, de vitellus et d'albumen exprimées en gramme par jour. Les premières sont toujours moins variables que les secondes respectivement. Le poids moyen de l'œuf et celui de l'albumen sont réduits significativement sous l'effet d'une déficience en méthionine, qu'elle soit ou non associée à un manque de protéine. Ces critères, peu variables, sont très sensibles. On peut se demander néanmoins s'ils sont spécifiques de la méthionine ou s'ils concernent également d'autres acides aminés. Le poids moyen du vitellus révèle en revanche une déficience en protéines totales

\section{TABLEAU 2}

Principaux résultats. Valeur moyenne des critères utilisés et ordre de grandeur des coefficients de variation (CV)

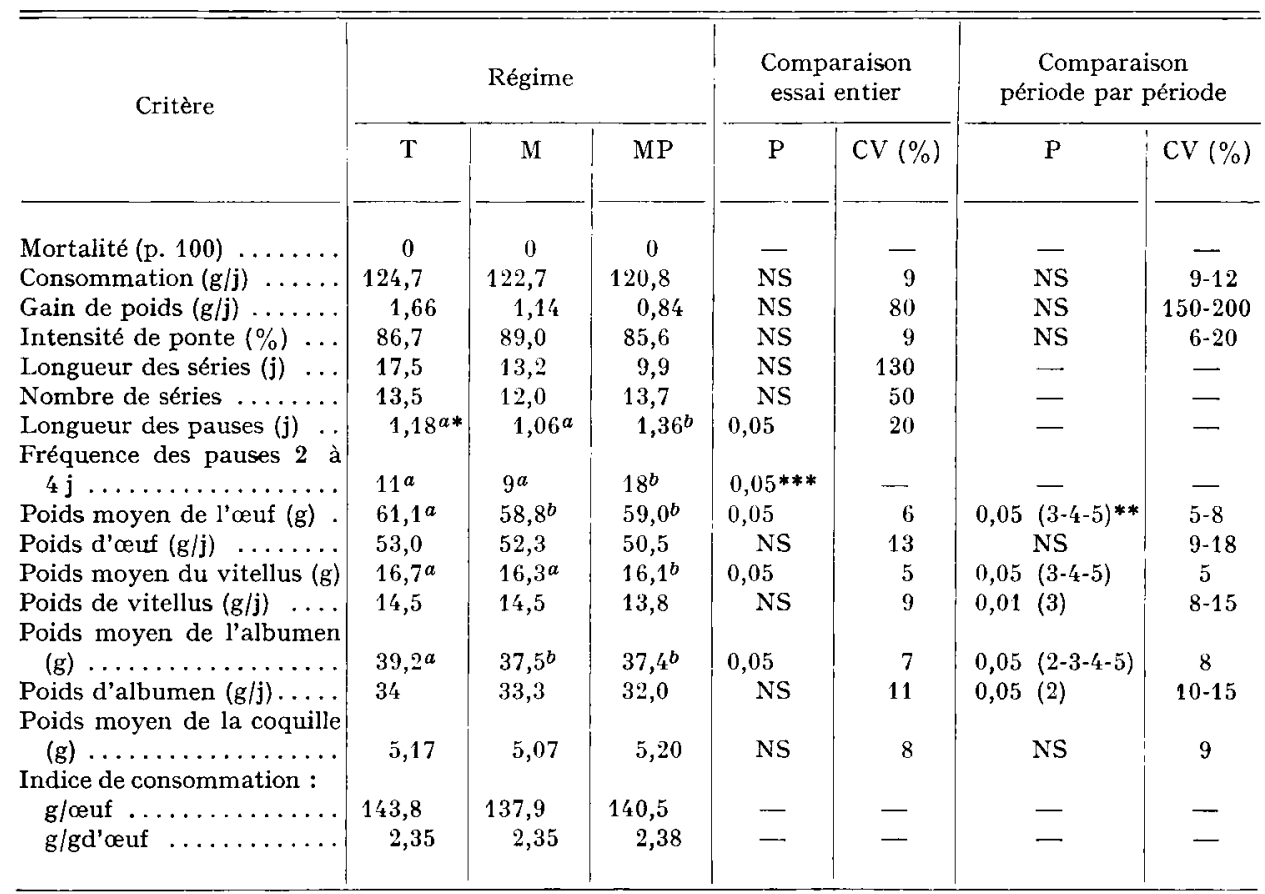

* Les nombres accompagnés de la même lettre ne diffèrent pas significativement entre eux.

** Les chiffres entre parenthèses indiquent les périodes pendant lesquelles la différence est significative.

*** Analyse par le critérium $\chi^{2}$.

Enfin, pour révéler les déficiences azotées du régime de la pondeuse, les différents critères n'ont pas la même signification selon l'époque et la durée du test. On distingue :

- Les critères tels que ceux relatifs aux pauses qui ne sont valables que pour l'essai entier.

- Les critères qui ont autant de valeur pour l'essai entier que pour une période de 28 jours (poids moyen de l'œuf et de ses constituants).

- Les critères qui ne peuvent être employés que pendant les 4 semaines qui suivent le pic de ponte : c'est le cas du poids du vitellus formé par jour. 
Cet essai confirme l'action distincte des protides totaux et de la méthionine sur les constituants de l'œuf (Moran, I969; Fischer, I969, Leclerco, I970 ; Larbier, Blum, Guillaume, I972). Plus originale est la mise en évidence du degré de variabilité des critères étudiés : les poids moyens de l'œuf, du vitellus et de l'albumen sont très peu variables d'un sujet à l'autre ; ils révèlent remarquablement les effets des divers facteurs nutritionnels. La variabilité augmente cependant en fin d'essai et la période privilégiée correspond au pic de ponte ou juste après. Enfin deux critères nouveaux - longueur moyenne des pauses et fréquences des pauses - se sont révélés intéressants et méritent de susciter des études à venir. Notons cependant que ces résultats ne sont valables que pour une souche donnée puisque plusieurs auteurs dont KRAUTMAN, I97I et AITKEN et al., I972 viennent de souligner les réponses imprévues et souvent opposées de souches diverses nourries d'un même aliment.

\section{Reçu pour publication en mai 1973.}

\section{SUMMARY}

\section{COMPARISON OF VARIOUS CRITERIA FOR ESTIMATING THE DIETARY PROTEIN VALUE IN LAYING HENS}

In order to determine the sensitivity of 17 different criteria accounting for laying hen production, an experiment was carried out on 3 groups of 24 hens each. The animals, of the type Rhode Island Red, were kept in houses with controlled environment for I 40 days, period during which the laying performances were the highest.

The two experimental diets, as compared to the balanced control diet, were slightly deficient in methionine only or in methionine and total protein.

The deficiency in methionine only effected the mean weights of the egg and of the albumen, which are very sensitive criteria. Total protein deficiency did not specifically result in a change either in the number of eggs layed or in the length of clutches. On the other hand, it resulted in a lowering of the mean weight of the vitellus. The length of the non laying periods and the frequency of the 2 to 4 days non laying periods represented original criteria, which seemed to be valuable to detect total crude protein deficiency of the diet.

The variability of the amounts of eggs, albumen and vitellus produced per day increased as the laying season progressed. It was always higher than that of the mean weights of the egg and of its constituents which remained constant during the whole trial. Thus, the specificity and sensitivity of the different criteria used to estimate the value of the dietary protein were not the same during each period of the experiment.

\section{RÉFÉRENCES BIBLIOGRAPHIQUES}

Aitken J. R., Biely J., Nikolaiczuk N., Robblee A. R., Summers J. D., Barr W. K., I972. Genotype $\times$ dietary protein level interactions in egg production stocks. Poult. Sci., 51, I578-1582.

Fisher C., I969. The effects of a protein deficiency on egg composition. Brit. Poult. Sci., 10, 149-154. Harms R. H., Waldroup P. W., 1963. Length of laying cycle as influenced by dietary protein level. Poult. Sci., 42, II95-II97.

Krautman B. A., I971. Genetic nutrient interactions in laying hens. Fed. Proc,, 30, I 8-I20.

larbier M., Blum J. C., Guillaume J., I972. Effets d'une déficience alimentaire en lysine et méthionine sur les performances de ponte et sur la teneur en acides aminés libres du jaune d'œuf. Ann. Biol. anim. Bioch. Biophys., 12, 125-138.

LECLERCQ B., r97o. Facteurs nutritionnels modifiant le poids de l'œuf et de ses constituants. Ann. Biol. anim. Bioch. Biophys., 10, 239-252.

Moran E. T., I969. Levels of dietary protein needed to support egg weight and laying hen production. Feedstuffs, 41 (22), 26 . -

Moreno R. S., Damron B. L., Wilson H. R., Harms R. H., r97o. Use of the laying cycle as a criterion of dietary protein or sulphur amino acid adquacy. Poult. Sci., 49, 1507-I 509 . 